\section{Determinantes de las exportaciones de Paraguay en el periodo 2000-2019}

\author{
Determinants of Paraguayan exports
}

in the period 2000-2019

\section{Luis D. Laino}

\section{RESUMEN}

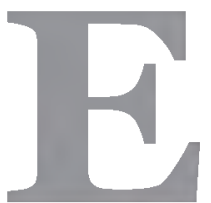

1 presente trabajo tiene como objetivo identificar determinantes de las exportaciones de Paraguay durante los veinte años del periodo 2000-2019 mediante un modelo gravitacional de comercio internacional. Con la aplicación de la técnica de datos de panel, se obtuvieron resultados que permiten afirmar que el $54 \%$ de las exportaciones paraguayas puede ser explicado por las variables incluidas en el modelo propuesto. La ecuación gravitacional especificada explicaría razonablemente determinantes de las exportaciones de Paraguay a los catorce países considerados, lo que permite algunas conclusiones relevantes.

Palabras clave: comercio internacional; modelo gravitacional; datos de panel.

\section{ABSTRACT}

The present work aims to identify determinants of Paraguayan exports during the twenty years of the period 2000-2019 through a gravitational model of international trade. With the application of the panel data technique, results allow us to affirm that $54 \%$ of Paraguayan exports can be explained by the variables included in the proposed model. The specified gravitational equation would reasonably explain determinants of Paraguayan exports to the fourteen countries considered, which allows for some relevant conclusions.

Keywords: international trade; gravitational model; panel data.
Luis D. Laino

ldlaino@chacoamericano.org

Centro de Investigación del Chaco Americano

Fundación Manuel Gondra PARAGUAY

COMO CITAR ESTE ARTÍCULO Laino, L. D. (2021). Determinantes de las exportaciones de Paraguay en el periodo 2000-2019. Revista de la Facultad de Ciencias Económicas, 26 (1), 99 - 113. http://dx.doi.org/10.30972/rfce.2615035

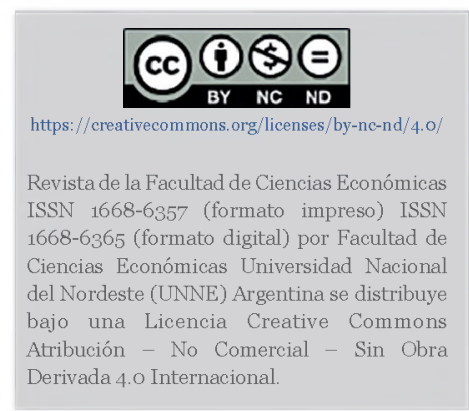




\section{INTRODUCCIÓN}

Las economías de los países están cada vez más interrelacionadas y esto se da a través de dos grandes vías: el comercio de bienes y servicios, y las finanzas. Mediante el comercio internacional, una parte de la producción de un país se exporta a otros, mientras que algunos bienes que se consumen en dicho país son producidos por otros países (Dornbusch \& Fischer, 1994).

Los vínculos comerciales son una de las formas en que la economía internacional afecta al crecimiento de un país. De acuerdo a Sachs \& Larraín (1994), en el debate de las estrategias de desarrollo para los países pobres, que viene de las décadas de 1950 y 1960, se identificaron dos patrones antagónicos: (i) la orientación hacia afuera, en la que un país abre sus mercados al resto del mundo y promueve sus exportaciones; y (ii) la orientación hacia dentro o sustitución de importaciones, cuando un país impone barreras significativas al comercio internacional y se centra en el desarrollo de la industria local para satisfacer el mercado doméstico.

Numerosos estudios documentaron el desempeño superior en términos de crecimiento económico que muestran los países orientados hacia afuera, y hallaron una alta correlación entre el crecimiento del producto global y el crecimiento de los ingresos por exportaciones. De esta forma, hay evidencia de que los países que consiguieron ampliar los mercados para sus exportaciones alcanzaron al mismo tiempo tasas más altas de crecimiento (Sachs \& Larrain, 1994).

Krugman \& Obstfeld (1995) afirman que las políticas de industrialización mediante la orientación hacia dentro o sustitución de importaciones no proporcionaron los beneficios esperados en crecimiento económico y nivel de vida, aunque tuvieron éxito en la promoción de industrias nacionales bajo la protección de aranceles y cuotas de importación. Por otro lado, resaltan que un pequeño grupo de países en vías de desarrollo logró su industrialización a través del desarrollo de la exportación de manufacturas, es decir, orientados hacia afuera, consiguiendo un rápido crecimiento en sus rentas y niveles de vida.

Teniendo en cuenta lo expuesto, es de relevancia identificar determinantes de las exportaciones de Paraguay que pudieran contribuir al diseño de estrategias de desarrollo mediante un mayor comercio internacional. Las exportaciones totales de Paraguay incluyen las exportaciones registradas por la Dirección General de Aduanas, las exportaciones de energía eléctrica, las reexportaciones y las otras exportaciones asentadas en la cuenta de bienes de la Balanza de Pagos, conforme clasificación del Banco Central del Paraguay (BCP). En 2019, las exportaciones registradas representaron el $62 \%$ del total, alcanzando preliminarmente USD 7.652 millones (BCP, 2019).

Considerando las exportaciones registradas en 2019 de acuerdo a sus niveles de procesamiento, las exportaciones de productos primarios, que incluyen cereales y granos de soja, fueron de USD 2.462 millones en 2019, mientras que las manufacturas de origen agropecuario, que abarca a la carne bovina, harina y aceite de soja, pieles y cueros, sumaron USD 2.623 millones. 
Por otra parte, las exportaciones de manufacturas de origen industrial, que incluye autopartes, materias plásticas y textiles, totalizaron USD 998 millones en el mismo año, y por último, las exportaciones de combustibles y energía fueron de USD 1.569 millones (BCP, 2019).

Durante 2019, los principales destinos de las exportaciones paraguayas fueron el Mercado Común del Sur, especialmente Brasil y Argentina, además de Chile, Rusia y otros países de Europa y Asia. En este sentido, las exportaciones a Brasil representaron el 33\% del total exportado, siendo los principales productos: energía eléctrica, autopartes y maíz. Las exportaciones a Argentina representaron el 23\% del total, destacándose granos de soja, energía eléctrica y autopartes; y las exportaciones a Chile fueron el $8 \%$ del total, siendo carne bovina, harina de soja y maíz los principales productos vendidos (BCP, 2019).

Durante los últimos veinte años, se aprecia un significativo aumento de las exportaciones paraguayas. Desde el año 2000, en que las exportaciones registradas sumaron USD $2.200 \mathrm{mi}-$ llones, se ha registrado un incremento promedio del 8,15\% anual. Considerando la importancia de la dinámica exportadora para elevar las tasas de crecimiento del producto y los niveles de vida, este trabajo tiene como objetivo identificar determinantes de las exportaciones paraguayas del periodo 2000-2019 a través de un modelo gravitacional de comercio internacional.

\section{EL MODELO GRAVITACIONAL DE COMERCIO INTERNACIONAL}

El modelo gravitacional de comercio internacional está basado en la ley física de gravitación universal planteada por Isaac Newton en su obra clásica "Principios matemáticos de la filosofía natural”, publicada en 1687. La ley de gravitación universal establece que la fuerza con que se atraen dos cuerpos debe ser proporcional al producto de sus masas dividido por la distancia entre ellos al cuadrado, con lo que a mayor masa y cercanía habrá más fuerza de atracción. Basado en este planteamiento, el economista William Reilly creó en 1931 un modelo gravitacional para medir el comercio minorista entre dos ciudades (Rosenberg, 2020).

En 1960, el economista Walter Isard y David Bramhall profundizan en los modelos gravitacionales en el libro "Métodos de análisis regional: una introducción a la ciencia regional" basándose, entre otras referencias, en trabajos de Carrothers, Carroll, Bevis, Dodd e Iklé de la década de 1950. Isard (1960) asevera que, si bien el modelo gravitacional está implícito en las obras de Carey, Ravenstein, Young y el ya citado Reilly, el primer gran impulso en su desarrollo proviene de los trabajos de Stewart y Zipf de 1948 y 1949.

Con respecto a la aplicación del modelo al comercio internacional, de acuerdo a Chaney (2011), fue el economista Jan Tinbergen quien en 1962 utilizó la analogía con la ley de gravitación universal de Newton para describir los patrones de los flujos agregados de intercambio 
bilateral entre países A y B como proporcionales a sus productos e inversamente proporcionales a la distancia que hay entre ellos.

De hecho, en la década de 1960, autores como Tinbergen, Poyhonen y Linnemann adaptaron el modelo gravitacional para la estimar el comercio entre países e introdujeron las variables básicas (Nascimento \& Júnior, 2013). Tinbergen analizó tres tipos de factores que explican los flujos comerciales: (i) la oferta potencial del país exportador y la demanda potencial del país importador, (ii) la población, y (iii) la resistencia al comercio, que a su vez puede ser natural o artificial. Se consideran barreras naturales a los costos y tiempo de transporte, mientras que artificiales a aquellas impuestas por los gobiernos, como ser tarifas, cuotas de importación y controles cambiarios (Sá Porto, 2002).

Krugman contribuyó también al perfeccionamiento del modelo gravitacional con la más difundida justificación teórica del modelo de los flujos bilaterales donde el comercio depende positivamente de la renta de los países y negativamente de la distancia entre los mismos presentada en 1980 (Kume \& Piani, 2000). Si bien no existen una completa correspondencia entre los principales modelos teóricos del comercio internacional y entre las variables utilizadas en el modelo gravitacional, varios autores demostraron que el mismo puede ser derivado tanto del modelo Heckscher-Ohlin como de modelos de competencia imperfecta (Azevedo, 2004).

De esta forma, la ecuación del modelo gravitacional, desarrollada inicialmente en la década de 1960 como una proposición empírica para explicar el intercambio comercial bilateral, fue fundamentada en posteriores años en una serie de artículos teóricos que demostraron que su formulación básica es consistente con varios modelos de flujos comerciales, con lo que su aplicación empírica se expandió para cubrir una variedad de aspectos (Frantianni, 2007).

El modelo gravitacional fortaleció su fundamentación teórica en las décadas de 1980 y 1990 con la incorporación de refinamientos estadísticos que mejoraron sus resultados y con el desarrollo de la nueva teoría del comercio internacional. El desarrollo del modelo gravitacional, sumado al aporte de la econometría y la introducción de la técnica de datos de panel, lo afirmaron como una fuerte herramienta de análisis de flujos de intercambio y medición de efectos de acuerdos comerciales entre países (Nascimento \& Júnior, 2013).

\section{LA TÉCNICA DE DATOS DE PANEL}

Con la aplicación de la econometría, los modelos gravitacionales pasaron de su configuración original de análisis transversal hasta el uso de datos de panel, lo que eliminó distorsiones que habían sido detectadas anteriormente (Nascimento y Júnior, 2013). La técnica de datos de panel es una combinación de datos de serie temporal y de corte transversal. 
De acuerdo a Baronio \& Vianco (2014), el principal objetivo de aplicar la técnica de datos de panel es capturar la heterogeneidad no observable, ya sea entre las unidades y en el tiempo, dado que esta heterogeneidad no se puede detectar con serie temporal o de corte transversal separadamente. Esta técnica permite realizar un análisis más dinámico al incorporar la dimensión temporal de los datos, lo que enriquece el estudio, particularmente en períodos de grandes cambios.

De esta forma, la aplicación de datos de panel permite analizar dos aspectos: (i) los efectos individuales específicos y (ii) los efectos temporales. Los efectos individuales son aquellos que afectan de manera desigual a cada uno de los agentes de estudio contenidos en la muestra (individuos, empresas, países), los cuales son invariables en el tiempo y afectan de manera directa las decisiones que tomen dichas unidades. Los temporales son los que inciden por igual en todas las unidades individuales del estudio y que pueden asociarse, por ejemplo, a los shocks macroeconómicos (Baronio \& Vianco, 2014).

La estimación de la regresión con la técnica de datos de panel puede ser realizada a través del modelo de regresión lineal bajo el método de Mínimos Cuadrados Ordinarios para datos combinados (MCOC), a través del modelo de Efectos Fịjos (EF) y mediante el modelo de Efectos Aleatorios (EA). La regresión de MCOC considera la muestra longitudinal sin tener en cuenta la naturaleza de corte transversal y de series de tiempo de los datos, por lo que conlleva un grave error de especificación al no considerar la heterogeneidad de los datos (Z. de Pintor, Batista de Oliveira, E. de Pintor \& Schneider, 2018).

Por su parte, en el modelo de EF el intercepto de cada individuo permanece fijo en el tiempo. Este modelo considera que existe un término constante diferente para cada individuo y supone que los efectos individuales son independientes entre sí. Con este modelo se considera que las variables explicativas afectan por igual a las unidades de corte transversal y que éstas se diferencian por características propias de cada una de ellas, medidas por medio del intercepto (Baronio \& Vianco, 2014).

El modelo de EA, en cambio, considera que los efectos individuales no son independientes entre sí, sino que están distribuidos aleatoriamente alrededor de un valor dado. Se asume que el número de factores que afectan a la variable dependiente, pero que no han sido incluidos explícitamente, pueden resumirse apropiadamente en la perturbación aleatoria. De esta forma, el modelo de EA considera que tanto el impacto de las variables explicativas, como las características propias de cada unidad de corte transversal, son diferentes. En el caso del modelo de EA no es aplicable el método de Mínimos Cuadrados Ordinarios debido a que no se cumplen sus supuestos, por lo cual es preferible la aplicación de Mínimos Cuadrados Generalizados (Baronio \& Vianco, 2014). 


\section{METODOLOGÍA: ESPECIFICACIÓN DEL MODELO PROPUESTO Y FUENTE DE DATOS}

La formulación del modelo gravitacional de comercio internacional se materializa en la ecuación gravitacional, que es uno de los hallazgos empíricos más robustos en economía internacional. La ecuación gravitacional establece que el intercambio bilateral entre dos países es proporcional a sus respectivos tamaños, medidos por el Productos Internos Bruto (PIB) de cada uno, e inversamente proporcional a la distancia geográfica que hay entre ellos (Chaney, 2011).

Anderson (1979), calificó a la ecuación gravitacional como el dispositivo comercial empírico más exitoso de los últimos años, afirmando que produce generalmente un buen ajuste, aplicada a una amplia variedad de bienes y factores que se mueven a través de fronteras regionales y nacionales en diferentes circunstancias.

La ecuación gravitacional básica es expresada de la siguiente manera y en forma logarítmica (Azevedo, 2004):

$M \ddot{i j}=B_{O}+B_{1} \ln Y i+B 2 \ln (Y i / N i)+B_{3} \ln Y j+B_{4} \ln \left(Y_{j} / \mathrm{Nj}\right)+B_{5} \ln D \ddot{i j}+e \ddot{i j}$

donde:

Mij es el comercio bilateral en exportaciones o importaciones nominales, o en la suma de ambas, del país i al país j;

$Y w$ es el PIB nominal de los países i y j;

$N w$ es la población de los países i y $\mathbf{j}$;

Dij es la distancia entre los países i y j;

$B 1, B 2, B 3$ y $B 4$ son parámetros que se espera tengan signo positivo;

$B 5$ es parámetro que espera tenga signo negativo; $y$

eij es el término de error.

Se espera que los coeficientes asociados al PIB de los países exportadores e importadores presenten signo positivo, debido a que cuanto mayor es la renta de un país, mayor será la variedad de productos disponibles para exportar y mayor será la preferencia de sus habitantes por la variedad de consumo.

Existen también razones que indican que el PIB per capita tiene un impacto positivo sobre el comercio, debido a que, en la medida en que los países se desarrollan, mayor es la oferta y la demanda de productos diferenciados. La distancia entre los países, por el contrario, representa una resistencia al comercio y tiene un impacto negativo (Azevedo, 2004). 
Dos formas estandarizadas de medir el tamaño de los países en un modelo gravitacional son el producto y la población. Manteniéndose constante el producto, el coeficiente de población es generalmente negativo, capturando el conocido fenómeno de que países más grandes tienden a ser relativamente menos abiertos al comercio como porcentaje de su producto. Es matemáticamente equivalente expresar las variables explicativas como PIB y PIB per capita, o como PIB y población, presentando signos inversos los coeficientes asociados a PIB per capita y población (Frankel, 1997).

Teniendo presente el objeto de este estudio, se especificó de la siguiente manera el modelo gravitacional a ser aplicado:

$\ln E X P i j=B O+B 1 \ln P I B i+B 2 \ln C A P i+B 3 \ln P I B j+B 4 \ln C A P j$

$+B 5 \ln D I S T i j+e \ddot{j}(2)$

donde:

EXPï es las exportaciones de Paraguay al país j;

$P I B i$ es el PIB de Paraguay;

$C A P i$ es el PIB per capita de Paraguay;

PIBj es el PIB del país j;

$C A P j$ es el PIB per capita del país j;

DISTij es la distancia entre la capital de Paraguay y la capital del país j;

$B 1, B 2, B 3 y$ y 4 son parámetros que se espera tengan signo positivo;

$B 5$ es parámetro que espera tenga signo negativo; y

eij es el término de error.

La ecuación 2. fue estimada con la técnica de datos de panel, a través del programa estadístico Gnu Regression, Econometrics and Time-series Library (Gretl), versión 2019c. Los datos abarcan a catorce países destino de exportaciones de Paraguay durante los veinte años del periodo comprendido entre 2000 y 2019. Los países considerados son: Alemania, Argentina, Brasil, Chile, Estados Unidos de América, España, India, Israel, Italia, Países Bajos, Perú, Rusia, Turquía y Uruguay. En conjunto, las exportaciones a estos catorce países representaron el $82 \%$ del valor total las exportaciones paraguayas del periodo analizado.

Para la estimación de la ecuación gravitacional se utilizaron datos anuales del periodo 2000-2019 separados en catorce paneles, uno por cada país importador. La fuente de los datos de exportaciones de Paraguay a estos destinos son estadísticas del Banco Central del Paraguay. Las cifras en dólares corrientes fueron convertidas a dólares constantes del año 2010.

Los datos de PIB y PIB per capita en dólares constantes del año 2010, y de extensión territorial en kilómetros cuadrados fueron obtenidos de la base de datos del Grupo Banco 
Mundial. Por último, los datos de distancia entre la capital de Paraguay y la capital de cada uno de los países importadores fueron obtenidos de www.distancecalculator.net

Para la determinación del modelo de datos de panel más adecuado se utilizaron los contrastes de Hausman y de Breusch-Pagan. Se realizó primeramente un análisis de las relaciones entre las variables para la detección de problemas de colinealidad, y un contraste para comprobar la normalidad del error, condición que debe cumplirse para la aplicación de los demás contrastes paramétricos de hipótesis asociadas al modelo de regresión lineal. Por otra parte, la estimación con datos de panel puede presentar problemas asociados a datos de corte transversal como la heterocedasticidad, y asociados a series temporales como la autocorrelación (Gujarati, 2006).

\section{RESULTADOS OBTENIDOS}

Aplicando la ecuación gravitacional propuesta fueron estimados tres modelos de datos de panel: (1) mediante Mínimo Cuadrados Ordinarios para datos Combinados (MCOC); (2) mediante Efectos Fijos (EF); y (3) mediante Efectos Aleatorios (EA). Se utilizaron datos anuales del periodo 2000-2019, con un total de 280 observaciones divididas en catorce paneles, un panel por cada país importador de productos de Paraguay.

Se realizó un análisis de las relaciones entre las variables del modelo a través de la matriz de coeficientes observándose una fuerte correlación entre el PIB y el PIB per capita de Paraguay, por lo que se excluyó la variable que mide PIB per capita de Paraguay de forma a evitar un problema de colinealidad. En el caso del modelo de EF se dio lo mismo además con la variable que mide distancia.

De acuerdo al contraste de normalidad de los residuos, el error tiene una distribución normal en los modelos de MCOC y de EA, al no rechazarse dicha hipótesis nula con un valor p no significativo al nivel del 1\%. Por el contrario, en el modelo de EF no puede asumirse una distribución normal. Se utilizó la estimación por desviaciones típicas robustas, de forma a evitar los problemas de heterocedasticidad y autocorrelación (Tabla 1.).

Seguidamente, con el objetivo de definir el modelo de datos de panel más apropiado fue aplicado el estadístico de contraste de Hausman, que resultó en un valor p no significativo al nivel del 1\%, indicación que permite aceptar la hipótesis nula de que el modelo de EA es consistente, en contra del modelo de EF. Por su parte, el multiplicador Lagrange de Breusch-Pagan arrojó un valor p significativo al nivel del 1\%, lo que implica una indicación en contra de la hipótesis nula de que el modelo MCOC es el adecuado, en favor del modelo de EA. 
Tabla 1. Resultados

\begin{tabular}{|c|c|c|c|}
\hline & Modelo 1: & Modelo 2: & Modelo 3: \\
\hline & MCOC & $\mathrm{EF}$ & $\mathrm{EA}(\mathrm{MCG})$ \\
\hline \multirow[t]{2}{*}{ const } & $-37,78^{* * *}$ & $-105,5$ & $-37,60^{* * *}$ \\
\hline & $(17,36)$ & $(89,48)$ & $(17,23)$ \\
\hline \multirow[t]{2}{*}{ ln_PIBi } & $2,297^{* * *}$ & 0,7754 & $2,179^{* * * *}$ \\
\hline & $(0,7323)$ & $(0,9194)$ & $(0,6530)$ \\
\hline \multirow[t]{2}{*}{ In_PIBj } & $0,4361^{* * *}$ & 5,041 & $0,5280^{\text {米获 }}$ \\
\hline & $(0,1783)$ & $(6,898)$ & $(0,1958)$ \\
\hline \multirow[t]{2}{*}{ ln_CAPj } & 0,02835 & $-3,386$ & 0,1257 \\
\hline & $(0,1149)$ & $(8,609)$ & $(0,1323)$ \\
\hline \multirow[t]{2}{*}{ In_DISTij } & $-1,267^{* * * * *}$ & & $-1,362^{\text {**** }}$ \\
\hline & $(0,2250)$ & & $(0,2102)$ \\
\hline $\mathrm{N}$ & 280 & 280 & 280 \\
\hline $\mathrm{R}^{2}$ & 0,5437 & & \\
\hline
\end{tabular}

Definido el modelo de EA como el apropiado, sus resultados indican que las variables independientes explican en general a la variable dependiente a un nivel del 54,37\% (valor R cuadrado). De esta forma, es posible afirmar que el PIB de Paraguay, el PIB y el PIB per capita de los países importadores, y la distancia entre la capital paraguaya y la de los países importadores explican el 54,37\% de las exportaciones paraguayas en el periodo 2000-2019.

El coeficiente de la variable PIB de Paraguay es estadísticamente significativo al nivel del $1 \%$ y presenta el signo positivo esperado, indicando que ante un aumento del $1 \%$ del PIB de Paraguay, las exportaciones paraguayas también se incrementarían en 2,18\%, manteniéndose constantes las demás variables del modelo (el valor p indica que la hipótesis nula de no significatividad individual debe ser rechazada). De igual manera, el coeficiente del PIB de los países importadores es significativo al nivel del 1\% y presentó el signo esperado, señalando que un aumento del $1 \%$ en la renta de los países importadores de productos paraguayos conllevaría un incremento de $0,53 \%$ en las exportaciones de Paraguay.

En cuanto a la variable PIB per cápita de los países importadores, su coeficiente no es estadísticamente significativo (el valor p indica que la hipótesis nula de no significatividad individual no puede ser rechazada). Pese a este resultado, el coeficiente presenta signo positivo conforme lo esperado de acuerdo a la teoría, señalando que un aumento de $1 \%$ en el PIB per cápita de los países importadores ocasionaría un incremento de 0,13\% en las exportaciones paraguayas. 
Por último, el coeficiente de la variable que mide la distancia entre la capital de Paraguay y las capitales de los países importadores es estadísticamente significativo y presenta signo negativo como indica la teoría. Su coeficiente expresa que un aumento de $1 \%$ en la distancia entre las capitales de Paraguay y los países importadores produciría una reducción de 1,36\% en las exportaciones paraguayas.

\section{DISCUSIÓN}

Las variables independientes del modelo gravitacional propuesto explican el 54,37\% de las exportaciones paraguayas, mediante la técnica de datos de panel y el modelo de Efectos Aleatorios (EA). Se destaca la fuerte relación positiva entre el PIB de Paraguay y las exportaciones a los catorces países compradores de productos paraguayos. El hecho de que una mayor renta del país aumente la capacidad de exportación implicaría que la mayor parte de los productos exportados no compiten en el mercado interno.

De acuerdo a Sachs \& Larrain (1994), entre los mecanismos que actúan en la relación entre exportaciones y mayor crecimiento del producto podría mencionarse al efecto del comercio sobre las economías de escala. En países en desarrollo como Paraguay, el tamaño del mercado doméstico es reducido, por lo que el comercio exterior lograría ensanchar el mercado a través de las ventas al resto del mundo.

En el mismo sentido, se observó una relación positiva del PIB y del PIB per capita de los países importadores de productos paraguayos y las exportaciones de Paraguay, lo que implicaría que estas exportaciones son absorbidas por el mercado interno de estos países, con lo que un aumento de la renta de los mismos tiene un efecto en la mayor demanda de exportaciones desde Paraguay.

Se verificó igualmente una fuerte relación, en este caso negativa, entre la distancia que separa a Paraguay de los países importadores y las exportaciones paraguayas, lo cual confirma la fundamentación teórica de que los costos y tiempo de transporte se constituyen en barreras naturales al comercio internacional.

A igual que otros países en vías de desarrollo, una parte importante del ingreso por exportaciones de Paraguay se origina en un reducido número de recursos naturales o de productos agrícolas. La dependencia respecto de estas exportaciones podría plantear un problema macroeconómico, ya que los precios de las materias primas son muy variables en comparación a los de los productos manufacturados. Al ser un país tomador de precios, los cambios drásticos en los ingresos por exportación producirían cambios en la renta real y en la cuenta corriente (Krugman \& Obstfeld, 1995). 
Del mismo modo, los precios de las exportaciones de los países en vías de desarrollo también podrían ser especialmente sensibles a las políticas macroeconómicas adoptadas por las economías industrializadas. Una reducción en la demanda agregada de los países industrializados se traduciría rápidamente en una caída de los precios de los productos y en un descenso de las rentas reales de los países en vías de desarrollo (Krugman \& Obstfeld, 1995).

\section{CONSIDERACIONES FINALES}

En primer término, se verificó la consistencia de los resultados con la fundamentación teórica del modelo gravitacional de comercio internacional, con una relación positiva entre las exportaciones paraguayas y el PIB de Paraguay, el PIB de los países importadores y el PIB per cápita de estos países. Por otra parte, se constató una relación negativa entre la distancia y las exportaciones.

Destacando estas relaciones y el reducido mercado interno de Paraguay, es posible señalar que una estrategia orientada hacia las exportaciones lograría aprovechar un potencial mercado, con su consecuente efecto de un mayor crecimiento económico. En este sentido, serían importantes las economías de escala de producción, pero también otros mecanismos que resultan de la competencia que mejora la productividad de las empresas locales y de la aplicación de políticas macroeconómicas eficientes.

En segundo término, debe considerase que las exportaciones de Paraguay son mayormente de productos primarios y manufacturas de origen agropecuario, siendo el país un tomador de precios internacionales. Esto implica una dependencia del mercado internacional, lo que podría ocasionar desequilibrios internos y externos en una economía en vías de desarrollo y de tamaño reducido. De esta forma, una caída en los precios de las exportaciones conllevaría el riesgo de una recesión económica.

Al respecto, sería relevante el análisis de ciertas medidas económicas asociadas a políticas de sustitución de importaciones, que en una fase inicial tuvieron éxito en la promoción de industrias nacionales y exportaciones de productos manufacturados de mayor valor agregado, cuyos precios internacionales son menos volátiles y podrían aportar a la diversificación de los productos de exportación.

Por último, sería igualmente importante la ampliación del estudio del comercio internacional de Paraguay centrado en sus productos más importantes, y en relación a la resistencia al comercio, la inclusión de variables que constituyen barreras artificiales al intercambio entre países, como tarifas y cuotas de importación, impuestas generalmente por países industrializados. 


\section{REFERENCIAS BIBLIOGRÁFICAS}

Anderson, J. (1979). A Theoretical Foundation for the Gravity Equation. The American Economic Review, Vol. 69, N. 1, 1979, pp. 106-116. American Economic Association.

Azevedo, A.. (2004). O efeito do Mercosul sobre o comércio: uma análise com o modelo gravitacional. Pesquisa e Planejamento Econômico, V. 34, No. 2, 2004. Disponible en:

http://ppe.ipea.gov.br/index.php/ppe/article/viewFile/71/45

Balgati, B. H. (2005). Econometric analysis of panel data. John Wiley \& Sons, UK.

Banco Central del Paraguay (BCP) (2019). Reporte de Comercio Exterior. Diciembre 2019, Asunción, Paraguay.

Baronio, A. \& Vianco, A. (2014). Datos de Panel. Departamento de Matemática y Estadística. Facultad de Ciencias Económicas, Universidad Nacional de Río Cuarto.

Chaney, T. (2011). The Gravity Equation in International Trade: An Explanation. University of Chicago, NBER and CEPR.

Dornbusch, R. \& Fischer, S. (1994). Macroeconomía. Sexta Edición. McGraw-Hill/Interamericana de España S.A. España.

Frankel, J. (1997). Regional trading blocs in the world economic system. Institute for International Economics. Washington, DC.

Frantianni, M.(2007). The Gravity Equation in International Trade. Handbook of International Business, 2nd Ed., Oxford University Press. Kelley School of Business. Indiana University.

Greene, W. (2003). Econometric Analysis. Fifth Edition. Prentice Hall. Upper Saddle River, New Jersey 07458.

Gujarati, D. N. (2006). Econometría Básica. Elsevier, Río de Janeiro.

Isard, W. (1960). Methods of Regional Analysis: An Introduction to Regional Science. Second Printing, 1962. The MIT Press. The Massachusetts Institute of Technology. 
Krugman, P. y Obstfeld, M. (1995). Economía Internacional. Teoría y Política. Tercera Edición. McGraw-Hill/Interamericana de España S.A. España.

Kume, H. \& Piani, G. (2000). Fluxos bilaterais de comércio e blocos regionais: uma aplicação do modelo gravitacional. Brasília: IPEA, Texto para Discussão, 749.

Nascimento, F. \& Júnior, D. (2013). A evolução do modelo gravitacional na economia. Revista Saber Humano, Recanto Maestro, N. 3, P. 163-175, 2013.

Rosenberg, M. (2020). Reilly's Law of Retail Gravitation. ThoughtCo. Disponible en: thoughtco.com/reillys-law-of-retail-gravitation-1433438

Sachs, J. \& Larraín, F. (1994). Macroeconomía en la Economía Global. Primera Edición. Prentice Hall Hispanoamericana S.A. México.

Zanquetta de Pintor, G., G. Batista de Oliveira, E. de Pintor \& M. Schneider (2018). Determinantes das exportações paranaenses para a América Latina e Caribe: Uma aplicação do modelo gravitacional. Revista Tecnologia e Sociedade, V. 14, N. 31, P. 189-207. ISSN: 1984-3526.

\section{CURRICULUM VITAE}

\section{Luis D. Laino}

Doctor en Ciencias Económicas por la Universidad Nacional de Asunción, Paraguay. Máster en Economía por la American University, Washington DC. Licenciado en Economía por la Universidad Católica de Asunción, Paraguay. Desempeñó funciones en la Secretaría Técnica de Planificación de Paraguay, y en la Universidad Nacional de Asunción como Profesor de la Facultad de Ciencias Económicas. Fue Encargado de Negocios-Jefe de Misión de Paraguay en Portugal y Embajador de Paraguay ante Cuba, concurrente ante Haití y República Dominicana. Actualmente, es asesor de empresas e investigador asociado al Centro de Investigación del Chaco Americano de la Fundación Manuel Gondra de Paraguay.

ldlaino@chacoamericano.org

REVISTA DE LA FACULTAD DE CIENCIAS ECONÓMICAS - UNNE - Argentina, Volumen 26 Núm. 1, enero-julio 2021, ISSN 1668-6365 http://revistas.unne.edu.ar/index.php/rfce 


\section{ANEXOS}

Coeficientes de correlación

\begin{tabular}{|lllllll|}
\hline ln_PIBi & ln_CAPi & ln_PIBj & ln_CAPj & ln_DISTij & $\ln \_E X P i j$ & \\
1,0000 & 0,9965 & 0,1166 & 0,1257 & $-0,0000$ & 0,3742 & ln_PIBi \\
& 1,0000 & 0,1149 & 0,1239 & 0,0000 & 0,3671 & $\ln \_C A P i$ \\
& & 0,3432 & 0,5383 & 0,0217 & $\ln \_P I B j$ \\
& & & 1,0000 & 0,2201 & 0,0237 & ln_CAPj \\
& & & 1,0000 & $-0,5453$ & ln_DISTij \\
& & & & 1,0000 & ln_EXPij \\
\hline
\end{tabular}

Valor crítico al 5\% (a dos colas) $=0,1173$ para $\mathrm{n}=280$

Modelo 1. MCO Combinados (MCOC)

\begin{tabular}{|lccccc|}
\hline & Coeficiente & Desv. Típica & Estadísticot & valor $p$ & \\
const & $-37,7819$ & 17,3635 & $-2,176$ & 0,0486 & $* *$ \\
ln_PIBi & 2,29661 & 0,732349 & 3,136 & 0,0079 & $* * *$ \\
ln_PIBj & 0,436092 & 0,178279 & 2,446 & 0,0294 & $* *$ \\
ln_CAPj & 0,0283547 & 0,114905 & 0,2468 & 0,8089 & \\
ln_DISTij & $-1,26739$ & 0,225024 & $-5,632$ & $<0,0001$ & $* * *$ \\
\hline
\end{tabular}

Variable dependiente: In_EXPij, desviaciones típicas robustas (HAC)

\begin{tabular}{|c|c|c|c|}
\hline Media de la vble. dep. & 18,65963 & D.T. de la vble. dep. & 1,677623 \\
\hline Suma de cuad. residuos & 358,3230 & D.T. de la regresión & 1,141487 \\
\hline R-cuadrado & 0,543667 & R-cuadrado corregido & 0,537030 \\
\hline $\mathrm{F}(4,13)$ & 17,29878 & Valor p (de F) & 0,000040 \\
\hline Log-verosimilitud & $-431,8331$ & Criterio de Akaike & 873,6662 \\
\hline Criterio de Schwarz & 891,8402 & Crit. de Hannan-Quinn & 880,9558 \\
\hline rho & 0,746144 & Durbin-Watson & 0,407511 \\
\hline
\end{tabular}

Contraste de normalidad de los residuos:

Hipótesis nula: [El error tiene distribución Normal]

Estadístico de contraste: Chi-cuadrado $(2)=8,11041$ con valor $\mathrm{p}=0,017332$

Modelo 2. Efectos Fijos (EF)

\begin{tabular}{|lcccc|}
\hline & Coeficiente & Desv. Típica & Estadísticot & valor $p$ \\
const & $-105,537$ & 89,4789 & $-1,179$ & 0,2593 \\
In_PIBi & 0,775395 & 0,919352 & 0,8434 & 0,4142 \\
ln_PIBj & 5,04142 & 6,89830 & 0,7308 & 0,4779 \\
ln_CAPj & $-3,38643$ & 8,60930 & $-0,3933$ & 0,7004 \\
\hline
\end{tabular}


Variable dependiente: In_EXPij, desviaciones típicas robustas (HAC)

\begin{tabular}{|lcll|}
\hline Media de la vble. dep. & 18,65963 & D.T. de la vble. dep. & 1,677623 \\
Suma de cuad. residuos & 251,5887 & D.T. de la regresión & 0,978065 \\
R-cuad. MCVF (LSDV) & 0,679596 & R-cuadrado 'intra' & 0,339143 \\
Log-verosimilitud & $-382,3236$ & Criterio de Akaike & 798,6473 \\
Criterio de Schwarz & 860,4387 & Crit. de Hannan-Quinn & 823,4319 \\
rho & 0,627118 & Durbin-Watson & 0,584890 \\
\hline
\end{tabular}

Contraste de normalidad de los residuos:

Hipótesis nula: [El error tiene distribución Normal]

Estadístico de contraste: Chi-cuadrado $(2)=31,6266$ con valor $\mathrm{p}=1,35635 \mathrm{e}-007$

\section{Modelo 3. Efectos Aleatorios (EA) (MCG)}

\begin{tabular}{|lcccc|}
\hline & Coeficiente & Desv. Típica & Estadístico $t$ & valor $p$ \\
const & $-105,537$ & 89,4789 & $-1,179$ & 0,2593 \\
$\ln \_\mathrm{PIBi}$ & 0,775395 & 0,919352 & 0,8434 & 0,4142 \\
$\ln \_\mathrm{PIBj}$ & 5,04142 & 6,89830 & 0,7308 & 0,4779 \\
$\ln \_\mathrm{CAPj}$ & $-3,38643$ & 8,60930 & $-0,3933$ & 0,7004 \\
\hline
\end{tabular}

Variable dependiente: In_EXPij, desviaciones típicas robustas (HAC)

\begin{tabular}{|lcll|}
\hline Media de la vble. dep. & 18,65963 & D.T. de la vble. dep. & 1,677623 \\
Suma de cuad. residuos & 365,9746 & D.T. de la regresi $\tilde{A}^{3} \mathrm{n}$ & 1,151519 \\
Log-verosimilitud & $-434,7912$ & Criterio de Akaike & 879,5824 \\
Criterio de Schwarz & 897,7564 & Crit. de Hannan-Quinn & 886,8720 \\
Rho & 0,627118 & Durbin-Watson & 0,584890 \\
\hline
\end{tabular}

Contraste de normalidad de los residuos:

Hipótesis nula: [El error tiene distribución Normal]

Estadístico de contraste: Chi-cuadrado (2) $=5,5585$ con valor $\mathrm{p}=0,0620849$

Contraste de Hausman:

Hipótesis nula: [Los estimadores de MCG son consistentes]

Estad. de contraste asintótico: Chi-cuadrado (2) = 4,89052 con valor $\mathrm{p}=0,0867037$

Contraste de Breusch-Pagan:

Hipótesis nula: [Varianza del error específico a la unidad = o]

Estad. de contraste asintótico: Chi-cuadrado $(1)=132,483$ con valor $\mathrm{p}=1,1732 \mathrm{e}-030$ 